\title{
Abordagens epistemológicas à Ciência da Informação: Fenomenologia e Hermenêutica
}

\author{
Epistemological approachs to the \\ Information Science: Phenomenology \\ and Hermeneutics
}

João Luiz Pereira MARCIANO'1

\begin{abstract}
RES UMO
Em sua patente interdisciplinaridade, a Ciência da Informação tangencia diversos domínios do conhecimento, quer estejam eles relacionados aos conteúdos de interesse, quer se relacionem às formas empregadas para representar tais conteúdos. Tal representação, por sua vez, é caracterizada não somente pelos símbolos e pelo vocabulário utilizados, mas principalmente pelo contexto intelectivo presente em sua elaboração e que acompanha todas as demais etapas do ciclo de vida da informação associada. Desse modo, a compreensão da mensagem (informação transmitida) e o conhecimento gerado a partir dela são dependentes tanto do aparato cognitivo do emissor quanto do receptor. O estudo desse processo requer um modelo capaz de analisar o conhecimento a partir de uma ótica isenta, independente do observador e do objeto de sua observação. A Fenomenologia propõe-se a realizar esse estudo do conhecimento do ponto de vista filosófico, interagindo, nesse processo, com as ciências cognitivas, as quais analisam os processos intelectivos envolvidos, e com a Ciência da Informação, a qual analisa a informação registrada.
\end{abstract}

Palavras-chave: Ciência da Informação; conhecimento; fenomenologia; ciências cognitivas.

\begin{abstract}
A B S TRACT
In its clear interdisciplinarity, the Information Science touches various knowledge domains, which are either related to its contents of interest, or related to the forms chosen to represent such contents. This representation, in turn, is characterized not only by its symbols and vocabulary, but mainly by the intelectual context available in its elaboration and which follows all the stages of the associated information life cicle. Therefore, the message (transmitted information) comprehension and the knowledge thus generated are dependent of both, the sender's and the receiver's cognitive apparatus. The study of this process requires a model capable of analyzing the knowledge from a neutral point of view, independent from both the observer and the object of its observation. The Phenomenology proposes to study knowledge from the philosophical point of view, interacting, in this process, with the cognitive sciences, which analyze the involved intelective processes, and with Information Science, which analyzes the registered information.
\end{abstract}

Key words: Information Science; knowledge; phenomenology; cognitive sciences.

\footnotetext{
1 Professor Doutor, Departamento de Ciência da Informação e Documentação, Faculdade de Economia, Administração, Contabilidade e Ciência da Informação e Documentação, Universidade de Brasília. Campus Universitário Darcy Ribeiro, Asa Norte, 70910-900, Brasília, DF, Brasil. E-mail:<marciano@unb.br>.

Recebido em 12/06/2006 e aceito para publicação em 19/09/2006.
}

TransInformação, Campinas, 18(3):181-190, set./dez., 2006 


\section{N T R O D U Ç Ã O}

Como ciência interdisciplinar, a Ciência da Informação permeia diversas áreas, sendo por elas influenciada e ainda carecendo de sólidos fundamentos epistemológicos. A análise dos problemas relacionados ao ciclo da informação é feita de modo multifacetado e fragmentado, com o uso de diversos métodos e metodologias que muitas vezes falham ao se reportar à sua base epistemológica e que ilustram uma dependência intrínseca, mesmo que involuntária, quanto às preferências pessoais ou coletivas dos autores, preferências que nem sempre são claramente expostas. A fim de elucidar o campo epistemológico com o qual lida a Ciência da Informação, apresentam-se a seguir algumas das abordagens apontadas na literatura.

Uma edição especial do Journal of Documentation, volume 61, número 1 de 2005, intitulada LIS and the Philosophy of Science, traz diversos artigos acerca das diferentes abordagens pelas quais a Ciência da Informação pode ser tratada, sob o ponto de vista epistemológico. Algumas das abordagens propostas são o realismo crítico, o neopragmatismo, a fenomenologia, o estruturalismo e o construtivismo social. Percebe-se uma grande variedade de possíveis epistemologias, não por coincidência em grande parte derivadas da filosofia da linguagem, sendo algumas conflitantes, que podem ser utilizadas em estudos da Ciência da Informação. Se, por um lado, isso corrobora em termos basilares a interdisciplinaridade atribuída a essa ciência, uma vez que lhe dá maleabilidade na escolha das ferramentas e recursos a serem utilizados, capacitando-a a imiscuir-se entre diversos domínios, fornecendo-lhes e deles obtendo suporte instrumental, por outro indica claramente a necessidade de uma melhor fundamentação dessa ciência sobre alicerces mais estáveis. Ao lado desse debate epistemológico, ocorre um outro pelo viés filosófico-ontológico, no qual os filósofos e os teóricos dos fundamentos da Ciência da Informação visam a identificar e caracterizar claramente as próprias bases filosóficas dessa ciência.

Neste sentido, Floridi (2002b) tenta delimitar o campo dos estudos filosóficos acerca da informação, iniciando por contextualizá-los historicamente, caracterizando-os como um novo campo e sugerindo que sejam abarcados pela expressão "filosofia da informação", definida por aquele autor nos seguintes termos:
Filosofia da informação é o campo filosófico que se dedica a: a) investigação crítica da natureza conceitual e dos princípios básicos da informação, incluindo sua dinâmica, utilização e ciências, e b) elaboração e aplicação de metodologias teóricas e computacionais da informação a problemas filosóficos. (FLORIDI, 2002b, p.137).

A dinâmica da informação apontada na primeira parte da definição acima diz respeito à constituição e à modelagem de ambientes de informação, ao ciclo da informação e à computação, tanto algorítmica quanto processual, privilegiando a informação sobre a computação, uma vez que esta não subsiste sem a pressuposição daquela. A segunda parte da definição, por sua vez, indica que a filosofia da informação agrega os requisitos de uma metodologia voltada a dar suporte aos estudos da Filosofia no que tange aos temas da informação. Em ambos os sentidos, a filosofia da informação lida com três tipos de domínios: tópicos (fatos, dados, problemas, fenômenos, observações), métodos (técnicas, abordagens) e teorias (hipóteses, explanações), visando a analisar diversos problemas. Floridi (2004b) aponta dezoito deles nos campos da análise, semântica e na própria natureza da informação (o que ela é e como se forma, por exemplo).

Duas abordagens à filosofia da informação são apontadas em Floridi (2003): uma analítica, voltada aos problemas conceituais e à lacuna de conceitos decorrentes da avalanche informacional experimentada nos últimos tempos; e outra metafísica, dedicada à inserção e à reinterpretação do Eu perante o mundo em transformação, construído virtualmente a partir de conceitos outrora eminentemente físicos. Outros conceitos relacionados à filosofia da informação, alguns aderentes à visão de Floridi, outros complementares, como a aplicação dos conceitos de Shannon (teoria da informação) e Wiener (cibernética) em uma abordagem mais pragmática aos problemas filosóficos da mente e da formação de significados (ADAMS, 2003), estão disponíveis, dentre outras fontes, em dois números especiais do periódico Minds and Machines, volume 13, número 4 de 2003 e volume 14, número 1 de 2004.

Também muito relevante à discussão acerca dos fundamentos epistemológicos da Ciência da Informação e da interação entre filosofia e informação é o número especial de um outro periódico, Library 
Trends - LIS and Philosophy, volume 52, número 3 de 2004, a incidência de artigos e particularmente de números especiais de periódicos de primeira linha acerca desses temas mostra a sua efervescência. Desse último periódico, dois artigos merecem consideração especial para a discussão tratada neste trabalho.

No primeiro deles, Cornelius (2004) questiona tanto a afirmação de Floridi (2002a) de que a Ciência da Informação é filosofia da informação aplicada, quanto o conceito de filosofia da informação por ele apresentado, que Cornelius considera "inocente acerca do caráter social" cumprido pela Ciência da Informação e dissociado dos aspectos práticos da área. No segundo artigo, Floridi (2004a) reafirma suas asserções, alegando que os aspectos práticos da Ciência da Informação não prescindem de uma embasada fundamentação teórica, ao mesmo tempo em que a avaliação dos conceitos acerca da informação em moldes filosóficos não exclui a aplicação de métodos e metodologias em estudos informacionais. Particularmente, Floridi considera tediosa a discussão sobre "o que é a informação", por não haver uma maneira simples de resolvê-la, preferindo considerar a questão "onde a informação está" - na mente ou no mundo? Em sua concepção, a informação encontra-se na interface entre o homem e o ambiente, como um limiar entre esses dois espaços - uma visão, de resto, claramente fenomenológica.

O debate acerca dos fundamentos da informação está longe de terminar. Na verdade, mal parece ter iniciado. Visando à adoção de uma abordagem humanista e social para os problemas relacionados à informação, tendo em vista os argumentos apresentados pelos autores acima citados e os vários estudos e propostas já realizados, como a sugestão feita por Wilson (2003) de empregar-se a fenomenologia como arcabouço de integração dos estudos da informação, apresentam-se uma postura fenomenológica, seus conceitos e métodos.

\section{As bases da teoria fenomenológica}

\section{Husserl}

A Fenomenologia representa uma dentre as diversas correntes filosóficas que se sedimentaram no decorrer do século XX, especialmente em sua primeira metade. Naquele período, as principais inquirições, conjeturas e publicações sobre o tema são devidas a Husserl e aos dois maiores expoentes existencialistas da Fenomenologia, Heidegger e Merleau-Ponty.

Edmund Husserl (1859-1938) preocupa-se com a perfeita caracterização do estado da mente consciente, identificado por ele como o elemento principal do ser, numa clara alusão à máxima cartesiana: "Penso, logo existo" (HUSSERL, 1996). Com esse objetivo, Husserl propõe rejeitar-se a aparente realidade do mundo (o conjunto das entidades físicas e perceptíveis), colocando-o "entre parênteses" (Einklammerung), método por ele denominado epoché fenomenológica: uma vez que o mundo e todas as suas entidades estão sempre presentes, quer sejam ou não experienciadas pelo observador, ele (o mundo) não deve interferir no processo de formulação do raciocínio, o qual, por sua vez, determina a realização da consciência. O que resta, após esse processo de distanciamento, é o selfque experimenta o mundo, estando a ele conectado pelo que Husserl chama de Ego Transcendental, o qual fornece razão e significado ao mundo e que existe independentemente da sua existência (MINGERS, 2001).

Para Husserl, o processo de indução fenomenológica compreende as seguintes etapas (FRAGATA, 1959):

- A análise "intencional" da relação entre o objeto como é percebido (noema) e a sua apreensão subjetiva (noesis); Husserl cunhou o termo "intencional" para representar a relação entre o objeto e sua aparência junto à consciência que o percebe, ou seja, seu significado;

- A epoché, conforme descrita anteriormente;

- A redução eidética (eidos = essência), o processo pelo qual se abstraem essências a partir da consciência e/ou da experiência, indo além dos padrões e estruturas convencionais de pensamento e ação a fim de identificar suas raízes comuns.

Desse modo, para Husserl o conhecimento não reside no observador nem tampouco no objeto observado, mas na concepção ou imagem do objeto formulada pelo observador. Husserl define a verdade como sendo a concordância perfeita entre o significado (formulado pelo observador) e o que é dado (o objeto), contextualizando o conhecimento como mais um dos fenômenos de estudo vistos por meio da epoché(STEGMÜLLER, 1977). 
Com essa formulação, Husserl influenciou grandemente a moderna teoria da consciência, com profundo impacto em ciências como a Sociologia (MYLES, 2004), a Psicologia tanto clínica (BURKITT, 2003) como organizacional (KARSSON; CHRISTIANSON, 2003) e a Administração (WHITE, 1990). Além disso, até o final de sua vida procurou manter uma postura crítica, mas equilibrada, acerca da ciência e da sua aplicação e desenvolvimento (HUSSERL, 1970).

De modo sucinto, segundo Sanders (1982), a pesquisa fenomenológica baseia-se em quatro grandes questões:

- Como o fenômeno ou experiência sob investigação pode ser descrito?

- Quais são os invariantes ou comunalidades, ou seja, os elementos comuns ou temas emergentes em tais descrições?

- Quais as possíveis reflexões acerca desses temas?

- Quais são as essências presentes nesses temas e reflexões?

Claramente, a fenomenologia se debruça sobre questões filosóficas envolvidas na geração do conhecimento e em sua aplicação, além de apresentar-se como uma epistemologia amplamente afeita a análises de fenômenos sociais e humanos. Nesse particular, a própria sociologia tem adotado a abordagem fenomenológica em contrapartida à abordagem clássico-científica ou normativa, no dizer de Wilson (1970), a qual se baseia na formulação e verificação de hipóteses.

O Quadro 1 apresenta as distinções apontadas por Sanders (1982) entre os paradigmas fenomenológico e normativo.

\section{Heidegger}

Martin Heidegger (1889-1976), para quem "um fenômeno é o que se mostra em si mesmo" (HEIDEGGER, 1985), estendeu ainda mais os limites da Fenomenologia. Enquanto Husserl entende a cognição como pensamento puro, Heidegger a vê como uma ação engajada, ao intuir que o homem, como ente auto-consciente, tem seu modo de ser caracterizado exatamente por sua forma de experimentar o mundo (HEIDEGGER, 1998; MINGERS, 2001). Em sua maior obra, Ser e Tempo, Heidegger delineia o que ele chama Dasein, traduzido como pré-sença (HEIDEGGER, 2002) ou ser-no-mundo (GEORGE, 2000), deixando clara sua preocupação com o ser humano como coletividade e não mais como indivíduo - sua preocupação, assim, não se restringe ao ser humano, mas abarca o ser consciente e inserido no mundo. Para Heidegger (1943), a essência da verdade consiste na liberdade de ser completo, ser e deixar ser.

A influência do pensamento de Heidegger estende-se desde a medicina e a enfermagem (DRAUCKER, 1999) até os sistemas de informação, sendo vista na obra de diversos autores. Suas discussões sobre a linguagem e a comunicação levaram à formulação de conceitos fundamentais, tais como (MINGERS, 2001):

- A cognição e o pensamento não são funções mentais isoladas; fazem, isto sim, parte das atividades do dia-a-dia, tornando-se essenciais ao ser-no-mundo.

- O conhecimento não consiste de representações de entidades objetivas independentes, formadas nas mentes dos indivíduos; ao invés disso, cada indivíduo realiza distinções, pelo uso da linguagem, no curso de suas interações com outros indivíduos, numa estruturação e reestruturação contínuas do mundo.

- A comunicação trocada em tais interações baseia-se na tradição e nas experiências pregressas, num complexo histórico dos agrupamentos estruturais formulados pelos indivíduos.

- A linguagem é a mais importante dimensão das ações do homem, mas ela deve ser vista como uma ação social por meio da qual o homem coordena suas atividades, mais que meramente como um veículo representativo e denotacional.

A influência de Heidegger é ainda maior ao se tratar dos estudos hermenêuticos do comportamento, conforme se verá mais adiante.

\section{Merleau-Ponty}

Maurice Merleau-Ponty (1908-1961) tem como uma de suas principais obras "Fenomenologia 
Quadro 1. Contrastes entre os paradigmas de pesquisa fenomenológico e normativo (adaptada de Sanders (1982)).

\begin{tabular}{|c|c|}
\hline Critério de diferenciação & Paradigma fenomenológico \\
\hline Apreensão do mundo & $\begin{array}{l}\text { O pesquisador enxerga o mundo como } \\
\text { indeterminado e problemático. Os fenô- } \\
\text { menos sob investigação são vistos mais } \\
\text { diretamente como resultantes de percepções, } \\
\text { intuição e significados pessoais. }\end{array}$ \\
\hline
\end{tabular}

Fenômenos investigados

Formulação do problema

Metodologia de pesquisa

Objetivo e inferências de pesquisa
Considera-se a "experiência vivida" pelos indivíduos. Considera tanto as características observadas como as qualidades específicas percebidas como formas pessoais de significado.

Inicia-se com uma atitude de epoché. Todos os preconceitos pessoais, crenças e afirmações sobre relações causais ou suposições são suspensas ou "colocadas entre parênteses”. Questões são formuladas e as respostas são analisadas.

Dá-se ênfase à descrição do mundo pelo ponto de vista das pessoas que o vivem e o experienciam. Todos os conceitos e teorias emergem dos dados da consciência, exigindo uma abordagem cognitiva que não pode ser replicada com exatidão.

Chegar a essências universais puras. A lógica da inferência é a comparação direta, resultando em novos insights ou reclassificações.

As generalizações dizem respeito apenas aos indivíduos específicos sob investigação. As conclusões servem como uma base de dados para investigações posteriores.
Paradigma normativo

O pesquisador vê o mundo como aproximadamente determinado ou não problemático. Escolhas pessoais ainda são necessárias para decidir quais características devem ser estudadas e como devem ser avaliadas.

Considera as características que são facilmente enumeráveis e empirica-mente verificáveis.

Inicia-se com uma hipótese de relação causal. A hipótese é verificada pela manipulação de uma ou mais variáveis independentes a fim de estudar o seu efeito sobre um comportamento específico (variável dependente).

Amplas generalizações abstratas ou teorias são aplicadas de uma forma lógico-dedutiva por meio das hipóteses das definições operacionais para formar um delineamento que pode ser replicado.

Interpretação estatística dos dados a fim de formular categorias ou normas. A lógica da inferência é a classificação e a serialização dos resultados, levando a comparações numéricas.

Generalizações são feitas com base na análise dos dados relativos a classes similares ou tendências universais que são expressas de um modo normativo (causa/ conseqüência, situação/ação, correlação). 
Também fundamental em Merleau-Ponty é o conceito de Embodiment ("a forma real e as capacidades inatas do corpo humano" (DREYFUS, 1996)), retomado por alguns autores (VARELA; THOMPSON; $\mathrm{ROSCH}, 1993$ ) em sua teoria da cognição atuante (enactive cognition), cujos dois principais aspectos são (MINGERS, 2001):

- A percepção consiste de ações guiadas de forma perceptiva (ou seja, a percepção de fatos anteriores influencia a percepção de fatos subseqüentes); e

- Novas estruturas cognitivas emergem dos padrões sensomotores que permitem à ação ser guiada pela percepção.

Desse modo, a atividade do organismo condiciona o que pode ser percebido num ambiente, e tais percepções, por sua vez, condicionam ações futuras. Deve-se acrescentar ainda que Merleau-Ponty integrou a análise fenomenológica à psicologia e à neurologia antes que uma definição formal das ciências cognitivas fosse apresentada como abarcando essas duas ciências (GALLAGHER; VARELA, 2001), numa associação que se vê cada vez mais aprofundada (BRUZINA, 2004), além de serem vistas grandes afinidades entre suas obras e as de outros autores sociais, como Searle e Bourdieu (MARCOULATOS; 2001, 2003).

\section{Ciência da Informação e Fenomenologia}

A interconexão entre a Fenomenologia e a Ciência da Informação mostra-se ainda mais evidente quando se observa que a primeira conceitua a linguagem como origem e expressão do conhecimento, ao passo que a última situa o documento, sua principal fonte de estudo, como veículo do conhecimento codificado e formalizado por meio da linguagem. Dessa forma, por meio da linguagem, ambas se contextualizam, uma quanto à gênese do conhecimento e a outra quanto à sua formalização.

Observa-se também que a percepção de si mesmo (auto-consciência) e do mundo é nitidamente um fenômeno informacional, ao mesmo tempo influenciando e sendo influenciado pelo contexto em que se insere o indivíduo. Essa concepção do conhecimento voltado à ação já fora apontada, no contexto da Ciência da Informação, por Wersig (1993), entre outros. Ao formalizar seu pensamento, o indivíduo externaliza suas percepções e associa a elas uma conotação pragmática, voltada a influenciar os comportamentos dos receptores daquela comunicação. Os receptores, por sua vez, terão sua percepção do mundo modificada pelo conhecimento recém-adquirido, percepção essa que dará origem a novas ações, num ciclo contínuo e renovado de geração e formalização do conhecimento. A efetividade dessas interações será tanto maior quanto mais intensa forem a produção e a busca pelo conhecimento no contexto analisado, ou seja, quanto mais ativo for o comportamento informacional dos indivíduos considerados.

Dessa forma, não causa estranheza que os comportamentos informacionais sejam outra área de proximidade entre a Fenomenologia e a Ciência da Informação, o que já fora apontado por, dentre outros, Wilson (1999) e Ng (2002), com especial destaque para a sociologia fenomenológica de Schutz, a qual encontra grande ressonância junto ao interacionismo simbólico de Blumer e que é influenciadora do "sense making"de Dervin (Wilson, 2002). Segundo a visão fenomenológica, o que se advoga aqui não é o uso das fontes de informação como mera redução de incertezas, mas sim a devida caracterização de problemas como sendo uma ruptura da concepção do mundo experimentada pelo observador: quando a percepção ou as atividades por ela encadeadas falham (quando, por exemplo, uma atividade de capacitação não surte o efeito desejado), surge um "problema" do ponto de vista fenomenológico (MINGERS, 2001). Em outras palavras, ocorre uma disparidade entre o objeto em observação e a sua imagem formulada pelo indivíduo.

Com vistas a sanar tal disparidade, os sistemas de informação, em vez de tentar impor um modelo estático e limitante - o que é uma prática usual -, devem ser então projetados e construídos de modo aberto e flexível, respeitando as particularidades de cada domínio e provendo o compartilhamento de significados e de experiências - enfim, de conhecimento. Desse modo, humanizam-se os sistemas, além de expandirem-se as fronteiras da organização, uma vez que por meio da comunicação realiza-se a troca de comportamentos (ações e percepções do mundo) entre os indivíduos, entre eles e as organizações e, por fim, entre as organizações vistas como sistemas. 
Um outro aspecto a salientar é o de que, além de importante ferramenta de análise de comportamentos, torna-se visível que a Fenomenologia pode cumprir o papel de suporte epistemológico, auxiliando a Ciência da Informação a situar-se perante outros domínios e a compreender melhor seus próprios objetos de estudo, bem como os métodos e as teorias que lhe dão embasamento, as disciplinas relacionadas e as interfaces adequadas entre usuários e sistemas.

Mas talvez a mais importante contribuição da Fenomenologia à Ciência da Informação, bem como a todas as outras ciências, seja a idéia de que não é possível ter uma percepção e uma observação "puras" do mundo. Sob essa concepção, similar à do realismo crítico, toda observação é dependente de uma teoria e de um contexto, estando continuamente sujeita a reformulações. As influências trazidas por essa visão de mundo estendem-se de Wittgenstein a Popper (MINGERS, 2004), com conseqüências fundamentais e permanentemente incidentes sobre o modo de produção científica ocidental.

\section{Hermenêutica}

Teóricos da Fenomenologia como Ricoeur (1975) e Gadamer (1998) propuseram um relaxamento crítico dos severos requisitos apresentados por Husserl para a epoché, indicando que é impossível interpretar qualquer ação social sem que se reporte a algum conhecimento e experiência prévios. Para os mesmos autores, a linguagem, em todas as suas modalidades, é o modo fundamental de ser-no-mundo (WHITE, 1990). Esse substrato lingüístico representa tanto o meio ou o instrumento pelo qual a comunicação se dá quanto, mais ainda, a abertura espaço-temporal compreendida como o ser-no-mundo (CAPURRO, 1982). É exatamente nesse contexto que se apresenta a hermenêutica, comumente (e simplificadamente) conhecida como a ciência da interpretação de textos, mas que vem sendo continuamente rediscutida e atualizada(VILLELA-PETIT, 2003), e que é cada vez mais utilizada em diferentes domínios (GEANELLOS, 2000), incluindo a Ciência da Informação (CAPURRO, 2000). A hermenêutica contrasta-se frontalmente com outras epistemologias, como o pragmatismo e o realismo.

Uma das principais críticas ao paradigma normativo é a sua incapacidade de considerar eventos e elementos em processos ditos whiteheadeanos ${ }^{2}$, ou seja, fenômenos que não se sucedem simplesmente, mas que se modificam de modo fundamental ao longo do tempo e de suas sucessivas interações. Desse modo, o paradigma normativo proporciona uma predição e uma explanação acuradas apenas para situações de interação particularmente rotineiras (DEETZ, 1973). O paradigma interpretativo, baseado na Fenomenologia, na Hermenêutica e na Filosofia da Linguagem, por outro lado, busca substituir o senso comum por uma visão mais essencial que possa tornar públicos interpretações e significados que passem despercebidos no dia-a-dia (DEETZ, 1973) (HEIDEGGER, 1985, p.188). Para tanto, três conceitos são fundamentais ao pesquisador (DEETZ, 1973):

- Implicação: considera-se o comportamento humano e seus produtos como a expressão de modos de ser-no-mundo. A ação humana, então, expressa-se ou implica um modo de existência particular, ou seja, o conjunto de possibilidades de uso encontradas em um mundo humano - as escolhas implicativas somente se tornam explícitas quando consideradas na relação entre o comportamento específico e o modo de existência em que se apresentam;

- Interpretação: representa o elo entre o comportamento expresso e o modo de existência, entre a ação e suas escolhas, ou entre um comportamento e suas implicações; é uma "leitura" do mundo implicado a partir do ato concreto. A fim de visualizar a interpretação de um comportamento, não se deve olhar para o objeto que o gera, mas sim para as implicações (possibilidades de uso) que ele expressa.

- Linguagem: a interpretação, assim como no paradigma normativo, pressupõe uma linguagem a priori, já intersubjetivamente cheia de significados (segundo DREYFUS, 1998, a linguagem tem o papel de chamar a atenção para algum aspecto do mundo já compartilhado) - a diferença consiste em que, enquanto o paradigma normativo considera a linguagem como sendo uma categoria residual que é

\footnotetext{
2 De Alfred North Whitehead, 1861-1947, matemático, logicista e filósofo britânico que, em companhia de Bertrand Russel, publicou os três volumes de Principia Mathematica, obra fundamental na lógica e metafísica do século XX.
} 
simplesmente acrescentada ao comportamento uma vez disposto em uma classe definida (categorização), a abordagem interpretativa sugere que a natureza (as possibilidades implicadas) de um fenômeno comportamental seja idêntica ao comportamento conforme sua nomeação - o fenômeno é o seu nome, e observar um comportamento nomeado é observar seu mundo humano e as possibilidades implicadas.

A Hermenêutica busca, desse modo, identificar um comportamento que expressa um mundo percebido e o entendimento do mundo que interpreta e explica o comportamento (DEETZ, 1973, p.150), em uma co-determinação que baseia o assim chamado "círculo hermenêutico" - um movimento de ir e vir entre idéias pré-concebidas (pré-conceitos) que são trazidas ao debate e os insights daí advindos (PIERCEY, 2004). Uma visão esquemática dessa rede de atuação está ilustrada na Figura 1.

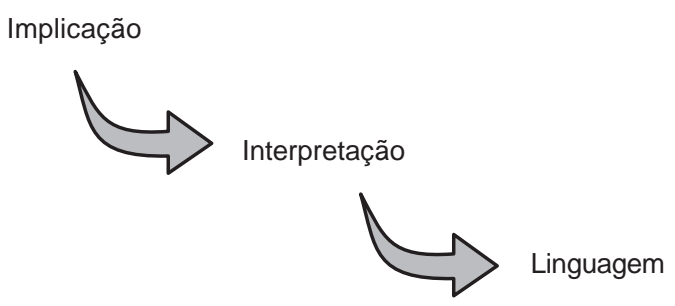

Figura 1. Rede de atuações observada no paradigma interpretativo.

Nota-se que a ação hermenêutica se dá com base na observação de comportamentos manifestos, escritos ou salientes, o que Ricoeur chama de "ação como texto" (RICOEUR, 1991, p.82). Ainda acerca desse mesmo tema, para Ricoeur (1990, p.33), o papel da Hermenêutica consiste na compreensão desses textos, mas "compreender um texto não é descobrir um sentido inerte que nele estaria contido, mas revelar a possibilidade de ser indicada pelo texto". O mesmo autor conceitua ainda a Hermenêutica como sendo a "teoria das operações de entendimento em sua relação com a interpretação de textos" (RICOEUR, 1982, p.43) e segue afirmando que "o objetivo da interpretação é produzir um discurso relativamente unívoco a partir de palavras polissêmicas e identificar esta intenção de univocidade na recepção das mensagens" (RICOEUR, 1982, p.44). Mais adiante, Ricoeur salienta ainda que a Fenomenologia permanece como a insuperável pressuposição da Hermenêutica, ao mesmo tempo que a Fenomenologia não pode se constituir sem uma pressuposição Hermenêutica (RICOEUR, 1982, p.101). Desse modo, Fenomenologia e Hermenêutica estão intrinsecamente associadas.

Questões acerca da corretude, plausibilidade ou verificação do entendimento embutido no paradigma interpretativo, segundo Deetz (1973, p.152), são irrelevantes, uma vez que a postura histórica do pesquisador é uma constituinte da natureza e das implicações do comportamento observado - o comportamento como descrito não pode ser visto sem a interpretação que Ihe foi dada. A fim de garantir-lhe objetividade e validade, alguns critérios metodológicos devem ser observados (DEETZ, 1973, p.153):

- Por princípio, o comportamento humano ou os seus produtos devem ser vistos como ações simbólicas, cuja natureza é composta pelas possibilidades implicadas e inseridas em um mundo sugerido por tais possibilidades.

- Os fenômenos humanos devem ser compreendidos na linguagem do mundo experiencial do qual fazem parte.

- Alguns critérios devem ser alcançados, tais como: Legitimidade: a interpretação atribuída é uma possibilidade na linguagem da comunidade? Correspondência: todos os comportamentos observados expressam o mesmo mundo implicativo? Apropriação de gênero: diferentes tipos de comportamento devem ser adequadamente interpretados de diferentes formas; Coerência: as implicações expressas são inteligíveis e plausíveis?

- O ponto de vista do indivíduo não deve ser literalmente considerado nem deve ser usado como critério para avaliar a interpretação - o comportamento objetivo expressa mais adequadamente o mundo de possibilidades implicadas.

- A intuição, quando vista como conhecimento essencial metodologicamente embasado, deve ser diferenciada da introspecção inferencial e do subjetivismo impressionista.

- Não se exige que o pesquisador se envolva diretamente no mundo sob escrutínio, mas que a inter- 
pretação-entendimento-explicação seja expressa na linguagem do mundo do comportamento observado a fim de se adequar e expressar de modo autêntico a estrutura de possibilidades implicativas.

No que diz respeito à Ciência da Informação, são usuais as interpretações segundo as quais um sistema de informações é composto pelo complexo de tecnologia (hardware software), enquanto outras dão um pequeno passo adiante ao abarcar a presença do usuário.

Contudo, conforme dito anteriormente, o usuário não é um indivíduo isolado - ele vive em determinado contexto (social ou organizacional, ao menos) e com ele interage, ao mesmo tempo influenciando-o e por ele sendo influenciado. A Fenomenologia, como epistemologia do conhecimento, tem muito a apresentar às diferentes análises dessa interação.

\section{REFERÊNCIAS}

ADAMS, F. The informational turn in philosophy. Minds and Machines, v.13, n.4, p.471-501, 2003.

BRUZINA, R. Phenomenology and cognitive science: moving beyond the paradigms. Husserl Studies, v.20, n.1, p.43-88, 2004.

BURKITT, I. Psychology in the field of being: Merleau-Ponty, ontology and social constructionism. Theory \& Psychology, v.13, n.3, p.319-338, 2003.

CAPURRO, R. Heidegger y la experiencia del lenguaje. 1982. Disponible en: <http://www.capurro.de/boss.htm>. Acceso en: 24 May 2005.

CAPURRO, R. Hermeneutics and the phenomenon of information. 2000. Available from: <http://www.capurro.de/ ny86.htm>. Acess: 24 May 2005.

CORNELIUS, I. Information and its philosophy. Library Trends, v.52, n.3, p.377-386, 2004.

DEETZ, S. An understanding of science and a hermeneutic science of understanding. The Journal of Communication, v.23, n.2, p.139-159, 1973.

DRAUCKER, C.B. The critique of heideggerian hermeneutical nursing research. Journal of Advanced Nursing, v.30, n.2, p.360-373, 1999.

DREYFUS, H.L. The current relevance of Merleau-Ponty's phenomenology of embodiment. Electronic Journal of Analytic Philosophy, v. 4, 1996. Available from: <http://www.focusing. org/apm_papers/dreyfus2.html>. Acess: 19 Apr. 2004.

DREYFUS, H.L. Why we do not have to worry about speaking the language of the computer. Information Technology \& People, v.11, n.4, p.281-289, 1998.

FLORIDI, L. On defining library and information science as applied philosophy of information. Social Epistemology, v.16, n.1, p.37-49, 2002.
CONSIDERAÇÕES FINAIS

A Ciência da Informação, como uma ciência mediadora entre outros domínios, percorre um longo arco desde a gênese até a disseminação e o uso da informação e do conhecimento gerado. Ao se dedicar à representação da informação, ela tangencia uma miríade de áreas, dentre as quais destaca-se a Fenomenologia. Como ciência filosófica, a Fenomenologia debruça-se sobre questões originárias desde a própria concepção do que seja o conhecimento até o seu papel como modificador da condição humana. Um imenso campo abre-se diante das duas ciências, onde a Ciência da Informação pode se valer do arcabouço da Fenomenologia para a sua própria compreensão e dos fenômenos que lhe são afeitos, incrementando sua inserção nos contextos científicos e sociais vigentes.

FLORIDI, L. What is the philosophy of information Metaphilosophy, v.33, n.1-2, p.123-145, 2002.

FLORIDI, L. Two approaches to the philosophy of information. Minds and Machines, v.13, n.4, p.459-469, 2003.

FLORIDI, L. LIS as applied philosophy of information: a reappraisal. Library Trends, v.52, n.3, p.658-665, 2004.

FLORIDI, L. Open problems on the philosophy of information. Metaphilosophy, v.35, n.4, p.554-582, 2004.

FRAGATA, J. A fenomenologia de Husserl como fundamento da filosofia. Braga: Cruz-Braga, 1959.

GADAMER, H.-G. The hermeneutic circle: the elevation of the historicity of understanding to the status of a hermeneutic principle. In: ALCOFF, L.M. (Ed.). Epistemology: the big questions. Malden, Massachusetts: Blackwell, 1998. p.232-247.

GALLAGHER, S.; VARELA, F. Redrawing the map and resetting the time: Phenomenology and the cognitive sciences. In: CROWELL, S.; EMBREE, L.; JULIAN, S. J. (Ed.). The reach of reflection. Delray Beach, Florida: Center for Advanced Research in Phenomenology, 2001.

GEANELLOS, R. Exploring Ricoeur's hermeneutic theory of interpretation as a method of analysing research texts. Nursing Inquiry, v.7, n.2, p.112-119, 2000.

GEORGE, V. The experience of being as goal of human existence: the Heideggerian approach. Washington: The Council for research in Values and Philosophy, 2000. (Cultural heritage and contemporary change. IIIB, South Asia, v. 2).

HEIDEGGER, M. On the essence of truth. 1943. Available from: <http://foucault.info/links/related-heidegger/heidegger. essenceOfTruth.eng.html>. Acess: 26 May 2005.

HEIDEGGER, M. Being and Time. Oxford: Basil Blackwell, 1985.

HEIDEGGER, M. The basic problems of the phenomenology. Bloomington: Indiana University, 1998. 
HEIDEGGER, M. Sere Tempo. 12.ed. Petrópolis: Vozes, 2002. V.1.

HUSSERL, E. The crisis of european sciences and transcendental phenomenology: an introduction to phenomenological philosophy. Evanston, Illinois: Northwestern University Press, 1970.

HUSSERL, E. Investigações lógicas: sexta investigação: elementos de uma elucidação fenomenológica do conhecimento. São Paulo: Nova Cultural, 1996.

KARLSSON, I.; CHRISTIANSON, S.A. The phenomenology of traumatic experiences in police work. Policing: An International Journal of Police Strategies \& Management, v.26, n.3, p.419-438, 2003.

MARCOULATOS, I. Merleau-Ponty and Bourdieu on Embodied Significance. Journal for the Theory of Social Behaviour, v.31, n.1, p.1-27, 2001.

MARCOULATOS, I. John Searle and Pierre Bourdieu: Divergent perspectives on intentionality and social ontology. Human Studies, v.26, n.1, p.67-96, 2003.

MERLEAU-PONTY, M. Fenomenologia da percepção. Rio de Janeiro: Freitas Bastos, 1971.

MINGERS, J. Embodying informations systems: the contribution of phenomenology. Information and organization, v.11, n.2, p.103-128, 2001.

MINGERS, J. Critical realism and information systems: brief responses to Monod and Klein. Information and organization, v.14, n.2, p.145-153, 2004.

MYLES, J.F. From doxa to experience: issues in Bourdieu's adoption of husserlian phenomenology. Theory, Culture \& Society, v.21, n.2, p.99-107, 2004.

NG, K.B. Toward a theoretical framework for understanding the relationship between situated action and planned action models of behavior in information retrieval contexts: contributions from phenomenology. Information Processing and Management, v.38, n.5, p.613-626, 2002.

PIERCEY, R. Ricoeur's account of tradition and the GadamerHabermas debate. Human Studies, v.27, n.3, p.259-280, 2004.

RICOEUR, P. Phenomenology and hermeneutics. Noûs, v.9, n.1, p.85-102, 1975.
RICOEUR, P. Hermeneutics and the human sciences. Cambridge: Cambridge University Press, 1982.

RICOEUR, P. Interpretação e ideologias. 4.ed. Rio de Janeiro: Francisco Alves, 1990.

RICOEUR, P. O si-mesmo como um outro. São Paulo: Papirus, 1991.

SANDERS, P. Phenomenology: a new way of viewing organizational research. The Academy of Management Review, v.7, n.3, p.353-360, 1982.

STEGMÜLLER, W. A filosofia contemporânea: introdução crítica. São Paulo: EDUSP, 1977.

VARELA, F.J.; THOMPSON, E.; ROSCH, E. The embodied mind: cognitive science and human experience. Cambridge, MA: MIT Press, 1993.

VILLELA-PETIT, M. Narrative identity and ipseity by Paul Ricoeur: from Ricoeur's 'Time and Narrative' to 'Oneself as an Other'. Online originals, 2003. Available from: <http://www. onlineoriginals.com/showitem.asp? itemID=286>. Acess: 4 May 2005.

WERSIG, G. Information science: the study of postmodern knowledge usage. Information Processing \& Management, v.29, n.2, p.229-239, 1993.

WHITE, J.D. Phenomenology and organization development. Public Administration Quarterly, v.14, n.1, p.76-85, 1990.

WILSON, T. Exploring models of information behaviour: the 'uncertainty' project. Information Processing and Management, v.35, n.6, p.839-849, 1999.

WILSON, T.D. Alfred Schutz, phenomenology and research methodology for information behaviour research. 2002. Available from: <http://informationr.net/tdw/publ/papers/ schutz02.html>. Acess: 24 May 2005.

WILSON, T.D. Philosophical foundations and research relevance: issues for information research. Journal of Information Science, v.29, n.6, p.445-452, 2003.

WILSON, T.P. Conceptions of interaction and forms of sociological explanation. American Sociological Review, v.35, n.4, p.697-710, 1970. 